\title{
A Simple and Fast Assay Based on Carboxyfluorescein-Loaded Liposome for Quantitative DNA Detection
}

\author{
Jacopo Sforzi, ${ }^{\dagger}$ Giuseppe Ferrauto, ${ }^{*}{ }^{\dagger} \odot$ Silvio Aime, ${ }^{\dagger, \dagger}$ and Simonetta Geninatti Crich ${ }^{\dagger \odot}$ \\ ${ }^{\dagger}$ Molecular Imaging Center, Department of Molecular Biotechnology and Health Sciences and ${ }^{\ddagger}$ IBB-CNR, c/o Molecular \\ Biotechnology Center, University of Torino, Via Nizza 52, 10126 Torino, Italy
}

\section{Supporting Information}

\begin{abstract}
The development of an innovative and easy way to run assays for the quantitative detection of DNA present in biological fluids (i.e., blood, urine, and saliva) is of great interest for early diagnosis (e.g., tumors) and personalized medicine. Herein, a new quantitative assay based on the use of highly sensitive carboxyfluorescein-loaded liposomes as signal amplification systems is reported. The method has been tested for the detection of low amounts of DNA sequences. The reported proof of concept exploits a target DNA molecule as a linker between two complementary oligonucleotides. One oligonucleotide is biotinylated at its $3^{\prime}$ end and binds to streptavidin-coupled magnetic beads, whereas the other one is conjugated to a cholesterol molecule incorporated in the phospholipidic bilayer of the fluorescent liposomes. In the presence of the target fragment, the correct formation of a construct takes place as witnessed by a strong fluorescence signal, amplified by dissolving lipidic nanoparticles with Triton X-100. The system is able to detect specific nucleotide sequences with a very low detection threshold of target DNA (tens of picomolar). The assay allows the detection of both single- and double-stranded DNA. Studies performed in human blood serum show the correct assembling of the probe but with a reduction of limit of detection (up to $\sim 1 \mathrm{nM}$ ). This liposome signal amplification strategy could be used not only for the detection of DNA but also for other nucleic acids (mRNA; microRNA) that are difficult to be quantified by currently available protocols.
\end{abstract}

\section{INTRODUCTION}

The access to new, fast, and low-cost molecular diagnostic platforms is crucial to enable more effective monitoring and personalized treatments in cancer patients. In fact, the rapid and accurate detection of genomic alterations is at the basis of both the early diagnosis and the selection of the correct targeted therapy. ${ }^{1}$ Moreover, in addition to neoplastic diseases, nucleic acids may represent potential markers for a wide array of pathologies, including metabolic, inflammatory, and infectious diseases. ${ }^{2}$ Despite the fact that standard protocols are still based on tissue biopsies to identify possible genomic alterations, these methodologies are limited by the difficulties related to the low accessibility of tumor tissue and by the risks associated with the repetition of many tissue sampling. ${ }^{3}$ Recently, the sampling of biological liquids (i.e., blood, serum, urine, and saliva) has been recognized as an alternative source of nucleic acids for diagnosis of diseases. ${ }^{1,4,5}$ In a liquid biopsy technique, great attention is devoted to the detection of the socalled circulating tumor DNAs (ctDNAs), a class of tumorderived DNAs that are not associated with cells and are fragmented in the bloodstream. ${ }^{6}$

In general, only very low amounts of ctDNA are present in healthy patients. Conversely, significant levels of ctDNA can be found in cancer. ${ }^{5,7,8}$ The methods routinely used for DNA/ RNA detection involve two steps, namely, (i) the in vitro amplification of the sequence of interest (by polymerase chain reaction, PCR, or reverse transcription PCR, RT-PCR) and (ii) the detection of target DNA/RNA by electrophoresis, southern/northern blots, sandwich hybridization assays, or next-generation sequencing. ${ }^{8,9}$ The most innovative and best performing methodologies are represented by digital droplet PCR (ddPCR) and beads, emulsification, amplification, and magnetics (BEAMing). Both of them show several advantages and disadvantages. ${ }^{10,11}$ The ddPCR methodology utilizes a droplet generator that allows the partition of single pieces of DNA into droplets using an oil/water emulsion. The routine detection of ctDNA is still hampered in the clinical practice not only for an intrinsic lack of sensitivity/specificity for ctDNA but also because the currently available procedures require a high level of expertise and a long execution time. ${ }^{12}$ Moreover, the facilities necessary for the analysis, as flow cytometry and sequencing machineries, are present in specialized and certified laboratories for this kind of testing but are not always available in ordinary diagnostic laboratories. ${ }^{13}$

Alternatively, there are many examples of sandwich hybridization protocols based on the use of highly sensitive radioactive probes ${ }^{14}$ or gold or fluorescent nanoparticles. ${ }^{15-17}$ The latter ones are preferred to the radioactive probes for

Received: May 20, 2019

Accepted: November 28, 2019 


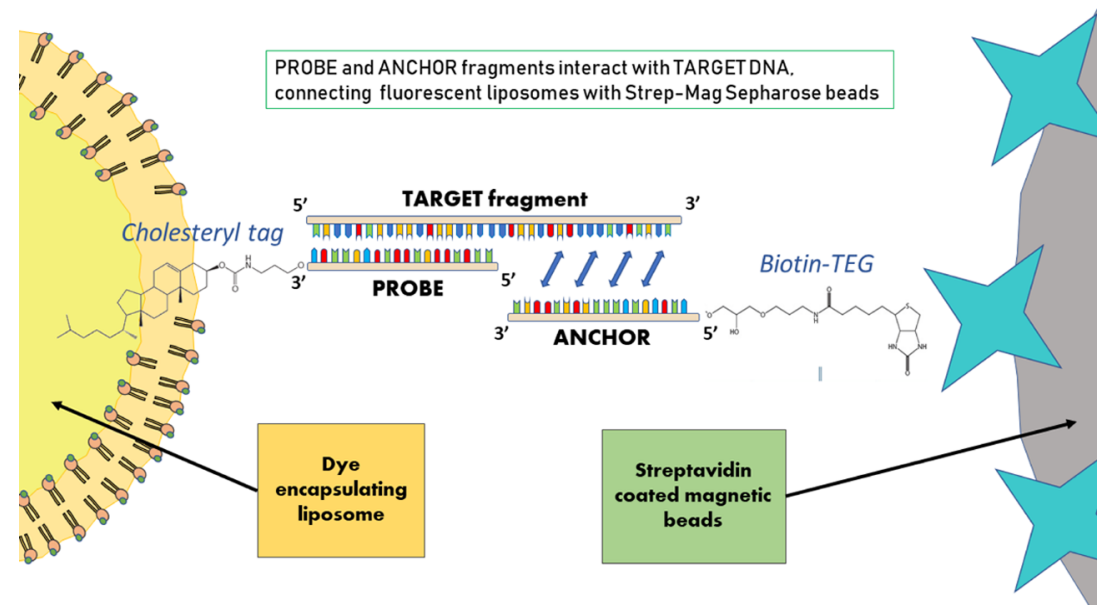

Figure 1. Assay proof of concept: step 1: Streptavidin Mag Sepharose beads interact with the biotinylated ANCHOR fragment. Step 2: 18 nucleotides of the ANCHOR fragment recognize specific complementary sequence on the TARGET fragment. Step 3: cholesteryl-tagged PROBE fragment, conjugated with $50 \mathrm{mM}$ CF liposome, interacts with the free $18 \mathrm{bp}$ long portion of the TARGET fragment.

obvious safety reasons, although they are less sensitive. ${ }^{18}$ Hence, the development of highly sensitive fluorescence-based methods for ctDNA detection may be attractive but challenging. In fact, this could help overcome the drawbacks of classical molecular biology assays (i.e., the need of a facility and expert staff $)^{19}$ without the need to use hazardous radioactive probes.

Useful routes to pursue the signal amplification may be provided by the use of nanoparticles. ${ }^{20}$ For example, fluorescein-encapsulating liposomes were reported to provide a 1000-fold decrease of limit of detection (LOD), with respect to fluorophore-labeled antibodies, in a sandwich hybridizationbased flow injection analysis system. ${ }^{21}$ Baeumner et al. proposed an assay based on dye-loaded liposomes, bearing oligonucleotides (complementary to the target DNA) on their outer surface and polyethersulfone membranes with streptavidin immobilized in the detection area. ${ }^{22}$ The target DNA of interest acts as a linker between the two shorter oligonucleotide capture probes. Upon loading high amounts of sulforhodamine B (SR-B) inside liposomal nanoparticles, the detection threshold was as low as $1 \mathrm{nM}$ analyte.

Starting from these results, herein, we propose a reproducible, versatile, and fast assay for DNA detection and quantification based on the use of highly stable liposomes loaded with fluorescent dyes that can be stored at $4{ }^{\circ} \mathrm{C}$ for 4 months without degradation. The proposed method does not require neither a high expertise level nor the use of radioactive molecules. It is based on the use of carboxyfluorescein (CF)loaded liposomes functionalized in the lipidic bilayer with a cholesterol-tagged DNA sequence, which is complementary to a portion of the target DNA.

The proof of concept of the proposed assay deals with the capture of both single- and double-stranded DNA (ssDNA and dsDNA, respectively) molecules using complementary oligonucleotides, exploiting the target ssDNA or dsDNA as a linker between two artificial strands. These strands are bound to dyeencapsulating liposomes through a cholesterol molecule and to streptavidin-coated magnetic beads via a biotin molecule. To observe the fluorescent signal from the high concentrated liposome payload, the lipidic nanoparticles were disrupted with Triton X-100 obtaining a high increase of signal intensity.

\section{RESULTS AND DISCUSSION}

Assay Description. The assay consists of the following four steps:

1) Commercially available magnetic beads functionalized with streptavidin molecules (Strep Mag) are incubated with a selected 18 base pair (bp) long ssDNA. This ssDNA, referred to as ANCHOR hereafter, was previously functionalized with a biotin-TEG group at its 5 ' end to bind streptavidin. The ANCHOR fragment was specifically designed to be complementary with the first 18 nucleotides present on the $3^{\prime}$ region of the fragment to be detected (referred to as TARGET hereinafter) (Figure 1).

2) Magnetic beads, covered with ANCHOR fragments, are incubated for $30 \mathrm{~min}$ with the TARGET containing solution. In the herein reported proof of concept, this fragment was designed to be $36 \mathrm{bp}$ long. After three washing steps with Hepes/ $\mathrm{NaCl}$ buffer, the unbound TARGET molecules and every other component possibly present in the matrix were washed out.

3) The $5^{\prime}$ portion of the TARGET fragments bound to the ANCHOR can recognize a third fragment hereinafter called PROBE. This third fragment, functionalized with a cholesterol moiety at its $3^{\prime}$ end, was previously intercalated in the phospholipidic bilayers of a liposome loaded with a highly concentrated solution of a fluorescent dye. The high concentration of the dye inside the liposome aqueous cavity causes an extensive "quenching" of the detectable fluorescence. ${ }^{23}$

4) After washing, to amplify the fluorescence signal, liposomes are destroyed with Triton X-100. The signal detected is directly proportional to the amount of the TARGET fragment bound to magnetic beads through ANCHOR.

Liposome Characterization. The used liposomes are prepared with $100 \%$ DPPC, a saturated phospholipid that ensures good membrane stability, avoiding leakage of the fluorescent molecule from the liposome core. The average diameter measured by dynamic light scattering (DLS) was of $110 \pm 31 \mathrm{~nm}$ with a PDI value of $0.106 \pm 0.007$. Among the commercially available dyes, CF was chosen due to its high solubility in water, its high quantum yield $(\Phi=0.92),{ }^{24}$ its low 
commercial cost, and the compatibility with a wide array of detectors commonly present in analytical laboratories. The maximum loading CF concentration was set at $50 \mathrm{mM}$. Higher CF concentrations were tested (e.g., 75 and $100 \mathrm{mM}$ ), but the resulting liposomal formulations, which display a PDI value higher than 0.5 , were not used because of the occurrence of high instability. When a liposome membrane was destabilized by Triton $\mathrm{X}-100$, the CF core content is released, and the fluorescent signal markedly increases (Figure 2A).

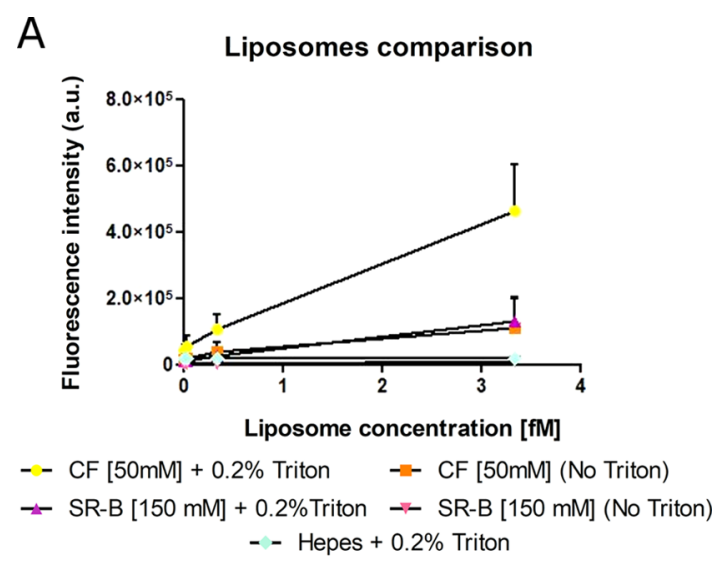

B

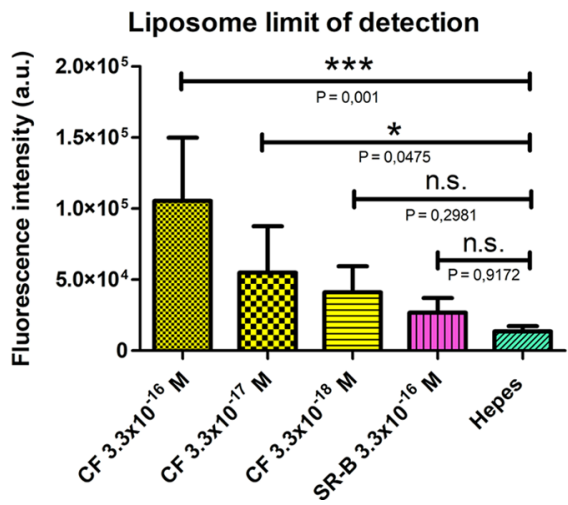

Figure 2. (A) Fluorescence intensity measured at increasing concentrations of SR-loaded $\left(\lambda^{\mathrm{ex}}=565 \mathrm{~nm}, \lambda^{\mathrm{em}}=583 \mathrm{~nm}\right)$ and CF-loaded liposomes $\left(\lambda^{\mathrm{ex}}=495 \mathrm{~nm}, \lambda^{\mathrm{em}}=517 \mathrm{~nm}\right)$ before and after the Triton X-100 addition. (B) Bar plot comparing fluorescence intensity at the lowest liposome concentrations. Error bars indicate standard deviation $(N=4)$.

CF-loaded liposomes where compared with liposomes of the same membrane composition but carrying up to $150 \mathrm{mM}$ sulforhodamine B (SR-B) inside their aqueous core. Liposomes loaded with $150 \mathrm{mM}$ SR-B have been previously reported as suitable probes for sandwich hybridization assays for nucleic acid markers. ${ }^{20}$ Figure 2 shows that a 3 -fold increase in fluorescence intensity was observed when $\mathrm{CF}$ liposomes were treated with Triton X-100. The concentration of the liposomal nanoparticles was determined by using the following equation

$$
[\text { liposomes }](\mathrm{nM})=\frac{[\mathrm{CF}]_{\mathrm{tot}} \times 3.97 \times 10^{8}}{[\mathrm{CF}]_{\mathrm{hydr}} \times(r-5)^{3}}
$$

Further information about this equation is reported in the Supporting Information.

Although sulforhodamine B-loaded liposomes showed a higher fluorescence increase when treated with Triton X-100, their minimum detectable liposome concentration was 2 orders of magnitude higher than the detection threshold determined for CF-loaded liposomes $\left(10^{-15}\right.$ for SR-B liposomes vs $10^{-17} \mathrm{M}$ for $\mathrm{CF}$ liposomes, as determined through subsequent dilutions in Hepes/ $\mathrm{NaCl}$ buffer; Figure $2 \mathrm{~B}$ ). Even though SR-B concentration inside liposomes is three times higher than $\mathrm{CF}$, the improved performance shown by CFloaded liposomes might rely on the different quantum yields of the internalized fluorescent compounds. ${ }^{18,25}$ These findings state that CF-loaded nanoparticles are more sensitive at lower concentrations, giving the possibility to investigate a very low amount of the target ssDNA and possibly detecting nucleic acids without the need of an amplification procedure. For these reasons, CF was used in the further experiments to set up the proposed assay.

The occurrence of a stable intercalation of cholesteryl-tagged ssDNA (PROBE) into the phospholipid bilayer was assessed using high-resolution nuclear magnetic resonance (NMR).

The specific peaks, characteristic of nucleotide sequences, appeared only in the ${ }^{1} \mathrm{H}-\mathrm{NMR}$ spectrum of liposomes incubated with the PROBE segment, while any nonspecific signals were detected in the control ones (Figure S1).

Moreover, the number of dialysis cycles needed to eliminate the nonencapsulated CF from liposome preparation was evaluated. It was reported that six dialysis cycles $(4 \mathrm{~h}$ at 4 ${ }^{\circ} \mathrm{C}$ for each cycle) are necessary (Figure S2). In concentrated suspension, the stability of liposomes has been assessed by measuring the fluorescence signal intensity of intact liposomes and Triton X-100-treated liposomes. When CF is released from liposomes, a fluorescence enhancement occurs (because of the "dequencing" of leaked CF). As reported in Figure S3, liposomes are stable up to 4 months.

Procedure Setup and Assay Detection Limit. The experimental setup takes less than $2 \mathrm{~h}$ (Figure S4). During the first $30 \mathrm{~min}$, magnetic beads, ANCHOR, and TARGET fragments were mixed to carry out the first annealing step. During the following $30 \mathrm{~min}$, incubation in the presence of the PROBE fragment allowed for the fluorescent labeling of the TARGET. Figure 3 shows the linear range of correlation between fluorescence intensity and TARGET concentration (Pearson correlation coefficient $=0.966$ ), with an LOD of $7 \times$ $10^{-11} \mathrm{M}^{26}$ The entire curve of fluorescence intensity versus

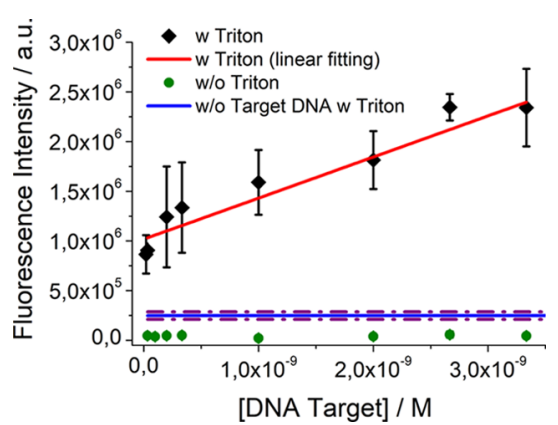

Figure 3. CF-loaded liposome fluorescence intensity measured after the addition of increasing TARGET concentrations to the Strep Mag and ANCHOR mixture. The blue line indicates the background fluorescence intensity measured without the TARGET (purple lines show standard error of the mean relative to the noise signal). The red line was obtained by linear fitting of the data. Error bars indicate standard deviation $(N=10)$. 
[DNA Target] is reported in Figure S5, and the parameters of the calibration curve are reported in Table S1.

After the washing steps, liposomes bound to Strep Mag beads through the DNA linkage showed a fluorescence intensity that is not distinguishable from the fluorescence signal of buffer (Figure 3, green dots). When treated with $0.5 \%$ Triton X-100, fluorescence intensity of the samples showed an increment directly correlated with the TARGET concentration (Figure 3, black diamonds). Few liposomes were able to bind to Strep Mag beads in a nonspecific manner when the TARGET fragment is not present, producing a low interference (Figure 3, blue line).

Next, the possibility to further improve the detection limit using microcuvettes was explored. The advantage of using microcuvettes relies on an $\sim 7$ times reduction of the final volume, thus allowing the increase of the dye concentration, which can allow the discrimination of lower concentrations of TARGET (e.g., $2 \times 10^{-11} \mathrm{M}$; Figure 4 ) not detectable by using the standard cuvettes (as shown by $P$ values obtained by using Dunnett's one-way ANOVA).

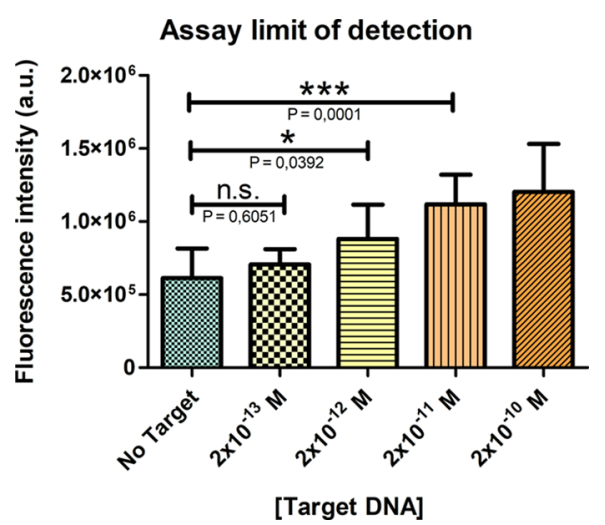

Figure 4. Microcuvettes assay. Error bars indicate standard deviation $(N=4)$.

The same experiment was repeated using SB-R-loaded liposomes to assess if the use of this dye makes it possible to reduce the background fluorescence signal. The results, as reported in Figure S6, confirmed the lower performance of this dye. In fact, the LOD was 2 orders of magnitude higher than that of CF-loaded liposomes $\left(2 \times 10^{-10} \mathrm{M}\right)$.

Figure 5 shows that SCRAMBLE fragments are not able to link the liposome and the magnetic components. In fact, the observed fluorescence signal was not distinguishable from the background in the absence of the TARGET. Furthermore, by maintaining the scramble- 36 and scramble- 100 in a $1: 1$ ratio and varying the TARGET molarity, no significant interference in the binding of TARGET to the ANCHOR fragment was noted, even when the SCRAMBLE:TARGET ratio was 1000:1. In fact, the linearity of the detection of the assay calibration curve did not vary.

Assay Behavior in the Presence of Double-Stranded TARGET. The assay response was also evaluated with double helix conformation of the TARGET fragment (dsTARGET). To obtain dsTARGET, the single-stranded TARGET was incubated with its complementary reverse fragment, heated to $85{ }^{\circ} \mathrm{C}$, and then let to cool at room temperature to favor the correct annealing of the two fragments. The formation of the dsDNA has been assessed trough agarose gel electrophoresis (Figure S7).
A

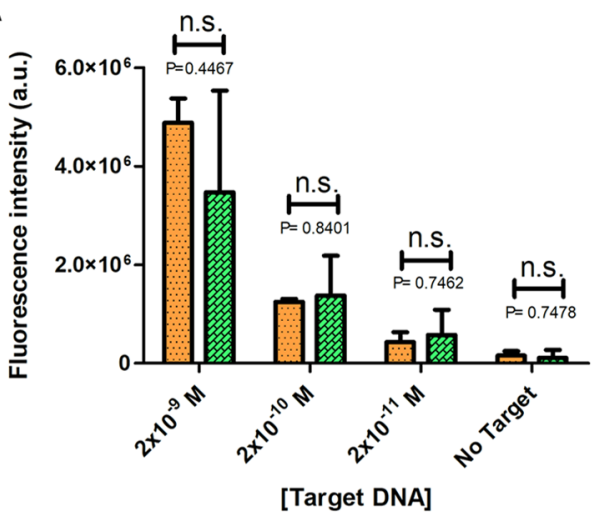

Target DNA + Scramble mix Target DNA only

B

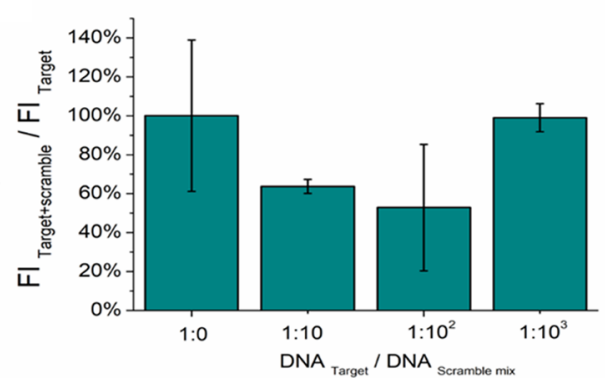

Figure 5. (A) Fluorescence intensity of the solution containing increasing amount of TARGET and a fixed $2 \times 10^{-8} \mathrm{M}$ scramble mix, added to the Strep Mag ANCHOR mixture. (B) TARGET concentration is normalized, and signal intensity of the solution obtained after incubation of TARGET alone is set to $100 \%$. Signal intensities in the presence of scramble mix are proportionally calculated and compared to the corresponding TARGET fluorescence signal. Error bars indicate standard deviation $(N=3)$.

To allow the assembly of the ANCHOR and PROBE components to the transient ssTARGET fragments, the samples were heated at $82{ }^{\circ} \mathrm{C}$ for $5 \mathrm{~min}$, and then, the temperature was rapidly decreased in liquid nitrogen for $10 \mathrm{~s}$. To avoid the annealing of the two complementary fragments and favor the binding of the assay components, the dsTARGET was incubated together with the ANCHOR and PROBE fragments in a 1:100 molar ratio. Finally, the liposome was added as the latest component due to its instability at high temperatures. No significant differences in signal intensity among the samples incubated at room temperature with ssTARGET and the ones incubated with dsTARGET were observed nor among the samples heated to $82{ }^{\circ} \mathrm{C}$ (Figure 6). Adding the Strep Mag beads after the rise in temperature did not alter the obtained signal intensity, thus suggesting that the overall decrease might be related to different causes and not the degradation of the magnetic beads (data not shown).

Assay Behavior in Serum. The effect of different biological matrices, among which is human serum, was tested with the aim of investigating the efficiency of the proposed DNA assay. The ssTARGET was dissolved in human serum before performing the already described assay. In serum, the assembly of the probe appears to be greatly hampered (Figure 7 and Figure S8), and the assay detection limit was 2 orders of magnitude higher, setting the LOD to $0.8 \times 10^{-9} \mathrm{M}$. These findings led us to investigate the possibility of unwanted interactions among serum and assay components, in particular, 
dsT assay investigation

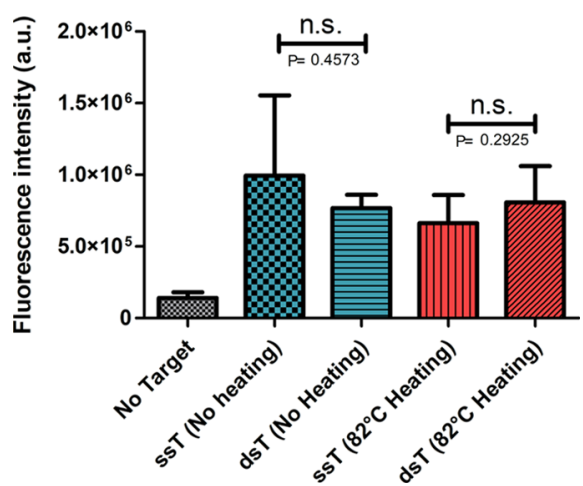

Figure 6. Comparison of assay behavior in the presence of doubleand single-stranded TARGET DNA (means are not significant among temperature-treated and not treated samples, $P$ value $>0.05)$. Error bars indicate standard deviation $(N=3)$.

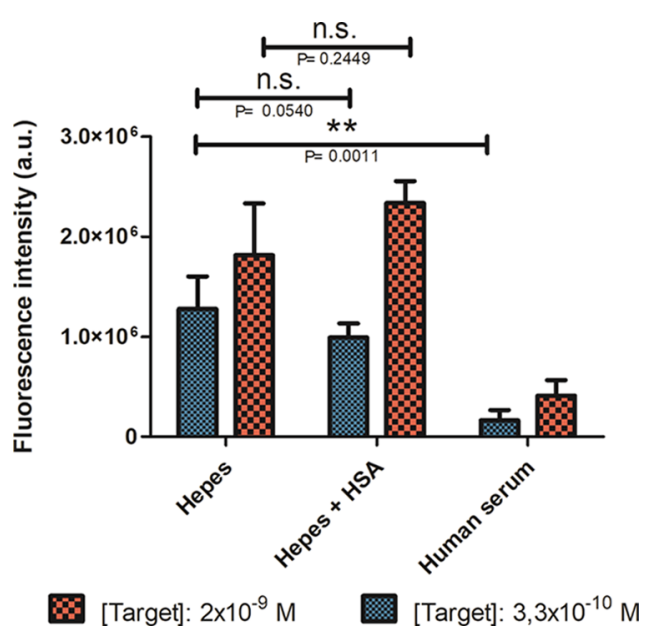

Figure 7. Assay behavior in Hepes, Hepes + HSA, and human serum: fluorescence intensity (a.u.) was measured after the addition of two concentrations of DNA TARGET $\left(3.3 \times 10^{-10}\right.$ and $\left.2.0 \times 10^{-9} \mathrm{M}\right)$ in three different media $(P$ value $>0.05)$. Error bars indicate standard deviation $(N=3)$.

with albumin, which is the most abundant serum protein. However, fluorescence measurements demonstrated that the presence of albumin did not affect the assembly of the components and the fluorescence signal intensity (Figure 7).

Further investigations were carried out to determine the role of phosphates on the decrease of assay performance (i.e., increased LOD) in serum. Thus, the assay components were assembled in PBS buffer prepared with 1 and $10 \mathrm{mM}$ phosphate concentrations. As seen from albumin, no significant decrease in the detected fluorescence intensity of the assay was observed, suggesting that phosphates do not play a role in the assembly of the deoxyribonucleic nor protein components (Figure S9). Finally, serum was inactivated through heating for $25 \mathrm{~min}$ at $55{ }^{\circ} \mathrm{C}$ and the assay performed as previously stated. No significant differences among not treated and treated sera have been observed, suggesting that the complement is not responsible for the decrease of the assay performance in this complex biological matrix (Figure S10). This can be due to the activity of DNase I, one of the components of the circulating free DNA (cfDNA) elimination system. $^{27}$

\section{CONCLUSIONS}

The herein described assay allows the detection of both singleand double-stranded DNA, measuring the signal arising from a fluorescent dye (carboxyfluorescein) encapsulated into liposomes at high concentration and released upon treatment with detergents (i.e., Triton X-100). The optimized liposomes are stable for a long period of time ( $\sim 4$ months) when stored at high concentration at $4{ }^{\circ} \mathrm{C}$. The LOD of target DNA is $7 \times$ $10^{-11} \mathrm{M}$, making it possible to investigate specific nucleotidic sequences at very low concentrations. Moreover, in comparison to multistep coupling procedures, the incorporation of a cholesteryl-modified ssDNA probe was allowed to obtain targeted liposomes without using expensive coupling reagents and time-consuming coupling procedures. ${ }^{21,28}$ Most of the commonly used techniques (among which are ddPCR and BEAMing) and sensors for DNA/RNA detection in liquid biopsies $^{29}$ have received an in-depth validation for clinical and preclinical applications. ${ }^{30,31}$ Compared to the these techniques, the herein proposed method shows the following advantages: (i) fast assay (execution time, approximately $2 \mathrm{~h}$ ); (ii) simplicity of execution, while both ddPCR and BEAMing need a medium-high level of expertise to be performed and require the presence of loader and reader machineries, which have an elevated price, whose purchase may not be justified for laboratories not fully dedicated to routinely screen for DNA markers; (iii) the decrease of biases derived from multiple amplifications of the low amount of starting nucleotidic material. Moreover, it is worth to mention the improvements with respect to the method reported by Baumner et al., ${ }^{22}$ namely, (i) a lower LOD (2 orders of magnitude); (ii) the use of a more efficient fluorescent dye (carboxyfluorescein); (iii) the use of Triton X-100 to obtain a fluorescence dequencing; and (iv) the use of magnetic beads instead of immobilized surfaces that have been proven to be very efficient for this kind of quantitative assays. ${ }^{32}$ As previously reported, plasma concentration values of cell-free DNA in melanoma and lung cancer patients are $\sim 36$ and $31 \mathrm{ng} / \mathrm{mL}$ plasma, respectively. ${ }^{33}$ The fragment length range is $134-144 \mathrm{bp}^{25}$ Thus, using 650 $\mathrm{g} / \mathrm{mol}$ as the average molecular weight for a base pair, we can theorize that the plasma cfDNA concentration range for these patients is $1 \times 10^{-10}$ to $6 \times 10^{-10} \mathrm{M}$. Considering that the fraction of mutant DNA ranges from 0.005 to $0.1 \%$ of the total DNA concentration in plasma, ${ }^{34}$ the expected amount of mutated DNA in serum is in the $10^{-13}$ to $10^{-15} \mathrm{M}$ range. In the case of colon carcinoma, the amount of mutant cfDNA increases dramatically, reaching a value of $\sim 8 \% .{ }^{11,35}$ This value is of the order of $10^{-11} \mathrm{M}$, that is, in the same order of magnitude of the LOD of the herein proposed assay, when the latter is performed in Hepes buffer. In principle, by exploiting a purification method to extract ctDNA from their biological matrices, it would be possible to detect and quantify nucleic acid markers without further amplification. In principle, the LOD of the herein reported method can be further reduced (i.e., enhanced assay performance) by using larger liposomes (e.g, medium unilamellar vesicles or giant liposomes), that is, containing a higher amount of fluorescent dye encapsulated in the inner core and/or by using a fluorescent dye with a higher quantum yield. Furthermore, other items have to be considered, especially for measurements in serum-containing specimens. First of all, the role of DNases in degradation of the target DNA has to be evaluated. For instance, serum DNA extraction protocols (based on phenol-chloroform extraction 
or pull-down techniques) can be employed to check whether this may contribute to the loss of sensitivity.

The herein reported method appears suitable, upon proper optimization, for the screening of circulating cancer DNA. The developed assay is versatile. In fact, by maintaining the proposed scheme (liposomes formulation, magnetic beads, etc.), it is possible to properly design the DNA sequences of ANCHOR and PROBE to specifically recognize TARGET sequences. Moreover, considering previously reported works, it appears possible to expand the herein reported method to the detection of longer ( $>36 \mathrm{bp}$ ) target DNA molecules. ${ }^{36,37}$

This is, in principle, applicable to the detection of DNA circulating in biological fluids (not only circulating tumor DNA). As an example, one may think of a number of pathogenic microorganisms that release their DNA in the bloodstream, thus opening the possibility to exploit their nucleic acids as markers for characterizing infectious diseases. Setting the nucleotidic sequence of the ANCHOR and PROBE fragments on a specific target sequence, the possibilities in the different markers detectable are limited only by the assay LOD.

Finally, future investigations could also lead to tune and validate the assay for the detection of RNAs, another class of diagnostic and prognostic markers rising in interest nowadays. ${ }^{38}$ This class of molecules is more difficult than DNAs to be analyzed since RNAs are more prone to enzymatic degradation and because of the complexity and biases present in RNA amplification protocols.

\section{EXPERIMENTAL SESSION}

Materials. DPPC (1,2-dipalmitoyl-sn-glycero-3-phosphatidylcoline) was purchased from Avanti Polar Lipids (Alabaster, Alabama, USA). Cholesterol, sodium chloride, sodium monobasic phosphate, sodium dibasic phosphate, potassium chloride, Hepes, chloroform, and all other chemicals were purchased from Sigma-Aldrich Inc. (Merck KGaA, Darmstadt, Germany). DNA oligonucleotides were purchased from Eurofin Genomics. Lyophilized human serum (Seronorm) was purchased from SERO AS, Norway.

Liposome Preparation. Large unilamellar vesicles (LUV) liposomes were prepared according to the classical thin lipid film hydration method as previously reported. ${ }^{39-41}$ Briefly, 45 mg of DPPC (1,2-dipalmitoyl-sn-glycero-3-phosphatidylcoline) were dissolved in a chloroform solution in a $250 \mathrm{~mL}$ glass balloon. The chloroform has been subsequently evaporated by using a rotavapor device in vacuum conditions at $60 \mathrm{rpm}$ at room temperature (RT). The lipid film formed after the solvent evaporation was left stirring for $1.5 \mathrm{~h}$ at $\mathrm{RT}$ to remove the presence of any water contamination. Subsequently, 1.5 $\mathrm{mL}$ of $50 \mathrm{mM}$ carboxyfluorescein solution, in Hepes buffer (0.15 M NaCl, $3.8 \mathrm{mM}$ Hepes, $\mathrm{pH} 7.4, \sim 320 \mathrm{mOsm} / \mathrm{L})$, was added to the lipid film. Then, it was heated through immersion in a water bath at a temperature of $56{ }^{\circ} \mathrm{C}$, which corresponds to the transition phase temperature of DPPC phospholipids. The bottom of the balloon was vortexed at max speed to ensure the detachment of the film from the glass wall of the recipient. These heating and vibrating steps were performed multiple times until the complete lipid film detachment. The formed solution was then introduced in an extruder device, heated at $56{ }^{\circ} \mathrm{C}$, and under argon pressure, the vesicles were forced to pass through a $400 \mathrm{~nm}$ filter membrane. After eight runs, the filter membrane was changed with a $200 \mathrm{~nm}$ one, and other eight runs were performed. To eliminate the unloaded CF molecules, the LUVs' suspension $(1.5 \mathrm{~mL})$ underwent extensive dialysis cycles against isotonic Hepes/ $\mathrm{NaCl}$ buffer solution $(2 \mathrm{~L})$ at $4{ }^{\circ} \mathrm{C}$ by using a $14 \mathrm{kDa}$ dialysis cellulose membrane. The external Hepes solution was replaced every $4 \mathrm{~h}$ for six cycles. After purification, the liposome size was determined by using dynamic light scattering (DLS) technology with a Zetasizer Malvern instrument. Liposomes were then stored at $4{ }^{\circ} \mathrm{C}$ in the absence of light to avoid photobleaching of the fluorescent molecules. ${ }^{42}$ Under these storage conditions, liposomes remained stable for at least 4 months.

Cholesteryl-DNA-Tagged Liposome Preparation. DPPC liposomes $\left(1 \times 10^{-8} \mathrm{M}\right)$ containing $50 \mathrm{mM}$ CF were diluted $1: 10$ in sterile Hepes buffer $(150 \mu \mathrm{L}$ of liposome solution in $1.35 \mathrm{~mL}$ of Hepes buffer). PROBE DNA was then added to this solution in a 300:1 PROBE/liposome ratio, and the resultant solution was incubated for $1.5 \mathrm{~h}$ on a shaking ThermoMixer at $4{ }^{\circ} \mathrm{C}$ in slow speed in the absence of light.

Subsequently, the solution was dialyzed overnight against 2 $\mathrm{L}$ of Hepes buffer in a $16 \mathrm{kDa}$ MWCO dialysis membrane at 4 ${ }^{\circ} \mathrm{C}$ in dark conditions to ensure the elimination of unbound PROBE molecules. After the dialysis, the functionalized liposomes were stored at $4{ }^{\circ} \mathrm{C}$ in dark conditions.

NMR Characterization. Liposome specimens $(20 \mu \mathrm{L})$ have been dissolved in $\mathrm{CHCl}_{3}$ into a $125 \mathrm{~mL}$ glass balloon. This procedure allows destroying liposomes and dissolving all the apolar molecules. Then, $\mathrm{CHCl}_{3}$ was evaporated using a rotavapor, and the specimen dissolved into deuterated methanol.

${ }^{1} \mathrm{H}$ NMR spectra of specimens have been acquired at $4{ }^{\circ} \mathrm{C}$ using a Bruker $600 \mathrm{MHz}$ spectrometer (AVANCE 600, 14 T). ${ }^{43}$

ssDNA Molecule Design. ssDNA molecules were purchased from Eurofin Genomics. A quality certificate describing yield and purification steps of the ordered oligonucleotide was delivered by the company together with the product. ssDNAs were artificially designed to be representatives of DNA sequences occurring in biological fluids. They have been designed through MeltCalculator, a Biophysics Integrated DNA Technologies online tool, through which the presence of self-dimers, hairpins structures, and annealing temperatures have been calculated.

ssDNAs used in our experiments have the following sequences:

TARGET: $5^{\prime}$ CTG GAC TGT TGA TTG TGG GAT TGA TAG GGC GAC TGC 3'.

ANCHOR: 5'-biotin-TEG-GCA GTC GCC CTA TCA ATC-3'.

PROBE: 5'-CCA CAA TCA ACA GTC CAGcholesterol-3'.

RT (reverse target): 5'-GCA GTC GCC CTA TCA ATC CCA CAA TCA ACA GTC CAG-3'.

S36 (scramble-36): 5'-AGC CGA TCT TAA TGG ATG GCC GCA GGT GGT ATG GAA-3'.

S100 (scramble-100): 5'-CAA CGG CAT GCG CAA CTT GTG AAG TGC CTA CTA TCC TTA AAC GCA TAT CTC GCA CAG TAA CTC CCC AAT ATG TGA GCA TCT GAT GTT GCC CGG GCC GAG T-3'.

Lyophilized ssDNAs were rehydrated with sterile TrisEDTA solution to obtain a final concentration of $100 \mu \mathrm{M}$. Purity of the shipped ssDNA samples was guaranteed by the 
manufacturer company and subsequently double-checked in our lab trough Nanodrop analysis.

Double-stranded TARGET DNA has been obtained through mixing the two complementary fragments TARGET and REVERSE TARGET in equimolar amounts, heating to $82{ }^{\circ} \mathrm{C}$, and letting it cure at room temperature to assure the correct annealing of these two ssDNAs.

Assay Protocol. Sterile Hepes buffer $(3 \mathrm{~mL})$ was added to a $15 \mathrm{~mL}$ Falcon tube, and $3 \mu \mathrm{L}$ of streptavidin-coated magnetic beads were added (Streptavidin Mag Sepharose, GE Healthcare S.P.A.). A $3 \mu \mathrm{L}$ ANCHOR ssDNA $100 \mu \mathrm{M}$ solution was added to this solution together with the desired amount of TARGET ssDNA. The Falcon tubes were placed on a shaker, and the beads and DNA mix were incubated for $30 \mathrm{~min}$ at room temperature to permit the binding of all the probe components. The tubes were then centrifuged for $2 \mathrm{~min}$ at $3000 \mathrm{rpm}$, and the magnetic beads were retained through a magnet. The solution was then eliminated together with all the unbound probe components, and three washing steps were performed using Hepes buffer. Cholesteryl-PROBE-tagged liposome $(100 \mu \mathrm{L})$ was added to the $3 \mathrm{~mL}$ Hepes buffer solution containing the beads and the components bound from the previous step. This solution was placed for $30 \mathrm{~min}$ on a shaker at medium speed and $4{ }^{\circ} \mathrm{C}$ in dark conditions. After this incubation, the tubes were again centrifuged for $2 \mathrm{~min}$ at 3000 rpm. Washing steps were performed to ensure that the elimination of all the elements was not bound to the magnetic beads until basal fluorescence intensity was comparable with the one present in Hepes buffer.

Triton X-100 (0.5\%; Sigma-Aldrich) was added to the liposome solution. After $15 \mathrm{~min}$, the total fluorescence intensity was analyzed with a spectrofluorometer (FluoroMax-4, Horiba Jobin Yvon). Spectrofluorometric excitation and emission parameters were set, respectively, at wavelengths of 492 and $517 \mathrm{~nm}$, wavelengths for carboxyfluorescein molecules, and at 565 and $586 \mathrm{~nm}$ for sulforhodamine B.

In the experiments performed in serum, Strep Mag beads, ANCHOR, and TARGET ssDNA were directly added to the serum (human serum, Seronorm SERO AS) and positioned on a shaker in slow speed at RT for $1 \mathrm{~h}$. Then, the samples were treated as described above with a centrifugation step, three washing steps in Hepes buffer, and the addition of DNAcholesterol-tagged liposomes.

Data Analysis and Statistic. Data have been obtained by using at least three independent experiments. The exact number of replicates has been inserted in each graph.

Data have been reported as mean \pm SD.

Pearson correlation coefficient of data in Figure 3 has been evaluated.

Unpaired two-tailed Student $t$ test has been carried out in Figures 5 and 6.

Dunnett's (one-way ANOVA) statistical test has been applied for the data in Figures $2 \mathrm{~B}$ and 4. One-way ANOVA test with Bonferroni correction has been applied for the data in Figure $7 .^{44}$

The following legend has been added to the figures: $* P$ value $<0.05$; $* * P$ value $<0.01$; and $* * * P$ value $<0.001$.

LOD has been calculated by using the following equation: $\mathrm{LOD}=3 \sigma / m$, where $\sigma$ is the SD of blank and $m$ is the slope of linear fitting of data in the linear range.

\section{ASSOCIATED CONTENT}

\section{Supporting Information}

The Supporting Information is available free of charge at https://pubs.acs.org/doi/10.1021/acsomega.9b01457.

Calculation of liposomes' concentration; NMR spectra of liposomes (Figure S1); assessment of the optimal number of dialysis cycles for purification of CF liposomes (Figure S2); assessment of liposomes stability over months (Figure S3); assessment of fluorescence intensity by using variable incubation time (Figure S4); CF-loaded liposome fluorescence intensity measured after the addition of increasing TARGET concentrations to the Strep Mag and ANCHOR mixture, entire dataset (Figure S5); DNA assay carried out by using $150 \mathrm{mM}$ sulforhodamine containing liposomes (Figure S6); 3\% agarose electrophoresis gel of TARGET DNA (Figure S7); calibration curve performed in human serum (Figure S8); comparison among DNA assay carried out in Hepes buffer, $1 \mathrm{mM}$ PBS buffer, and $10 \mathrm{mM}$ PBS buffer (Figure S9); comparison among DNA assay carried out in Hepes buffer, in normal serum, and in inactivated serum (Figure S10); calibration curve parameters (Table S1); comparison of LOD by using buffer, serum or buffer, and microcuvettes (Table S2); and supplementary references (PDF)

\section{AUTHOR INFORMATION}

\section{Corresponding Author}

*E-mail: giuseppe.ferrauto@unito.it.

ORCID

Giuseppe Ferrauto: 0000-0003-4937-6140

Simonetta Geninatti Crich: 0000-0003-2998-5424

\section{Author Contributions}

The manuscript was written through contributions of all authors. All authors have given approval to the final version.

Notes

The authors declare no competing financial interest.

\section{ACKNOWLEDGMENTS}

This project has received funding from the European H2020INFRADEV-2014-2015 project CORBEL (grant no. 654248).

\section{ABBREVIATIONS}

BEAMing, beads, emulsification, amplification, and magnetics; bp, base pair; CF, carboxyfluorescein; cfDNA, circulating free DNA; ctDNA, circulating tumor DNA; ddPCR, digital droplet PCR; DLS, dynamic light scattering; DPPC, 1,2-dipalmitoylsn-glycero-3-phosphatidylcoline; dsTARGET, double-stranded TARGET; LUV, large unilamellar vesicles; NMR, nuclear magnetic resonance; PCR, polymerase chain reaction; rpm, rotation per minute; RT, room temperature; RT-PCR, reverse transcription PCR; SR-B, sulforhodamine B; ssDNA, singlestranded DNA; ssTARGET, single-stranded TARGET

\section{REFERENCES}

(1) Butler, T. M.; Spellman, P. T.; Gray, J. Circulating-tumor DNA as an early detection and diagnostic tool. Curr. Opin. Genet. Dev. 2017, $42,14-21$.

(2) Sampson, D. L.; Fox, B. A.; Yager, T. D.; Bhide, S.; Cermelli, S.; McHugh, L. C.; Seldon, T. A.; Brandon, R. A.; Sullivan, E.; Zimmerman, J. J.; Noursadeghi, M.; Brandon, R. B. A Four-Biomarker 
Blood Signature Discriminates Systemic Inflammation Due to Viral Infection Versus Other Etiologies. Sci. Rep. 2017, 7, 2914-2931.

(3) Chi, H.; Hansen, B. E.; Tang, W. Y.; Schouten, J. N. L.; Sprengers, D.; Taimr, P.; Janssen, H. L. A.; de Knegt, R. J. Multiple biopsy passes and the risk of complications of percutaneous liver biopsy. Eur. J. Gastroenterol. Hepatol. 2017, 29, 36-41.

(4) Leon, S. A.; Shapiro, B.; Sklaroff, D. M.; Yaros, M. J. Free DNA in the serum of cancer patients and the effect of therapy. Cancer Res. 1977, 6, 646-650.

(5) Crowley, E.; Di Nicolantonio, F.; Loupakis, F.; Bardelli, A. Liquid biopsy: monitoring cancer-genetics in the blood. Nat. Rev. Clin. Oncol. 2013, 10, 472-484.

(6) Heitzer, E.; Ulz, P.; Geigl, J. B. Circulating tumor DNA as a liquid biopsy for cancer. Clin. Chem. 2015, 61, 112-123.

(7) Forshew, T.; Murtaza, M.; Parkinson, C.; Gale, D.; Tsui, D. W. Y.; Kaper, F.; Dawson, S.-J.; Piskorz, A. M.; Jimenez-Linan, M.; Bentley, D.; Hadfield, J.; May, A. P.; Caldas, C.; Brenton, J. D.; Rosenfeld, N. Noninvasive identification and monitoring of cancer mutations by targeted deep sequencing of plasma DNA. Sci. Transl. Med. 2012, 4, 136ra68-136ra68.

(8) Kloten, V.; Rüchel, N.; Brüchle, N. O.; Gasthaus, J.; Freudenmacher, N.; Steib, F.; Mijnes, J.; Eschenbruch, J.; Binnebösel, M.; Knüchel, R.; Dahl, E. Liquid biopsy in colon cancer: comparison of different circulating DNA extraction systems following absolute quantification of KRAS mutations using Intplex allele-specific PCR. Oncotarget 2017, 8, 86253-86263.

(9) Akca, H.; Demiray, A.; Yaren, A.; Bir, F.; Koseler, A.; Iwakawa, R.; Bagci, G.; Yokota, J. Utility of serum DNA and pyrosequencing for the detection of EGFR mutations in non-small cell lung cancer. Cancer Genet. 2013, 206, 73-80.

(10) Baker, M. Digital PCR hits its stride. Nat. Methods 2012, 9, 541-544.

(11) Diehl, F.; Li, M.; Dressman, D.; He, Y.; Shen, D.; Szabo, S.; Diaz, L. A.; Goodman, S. N.; David, K. A.; Juhl, H.; Kinzler, K. W.; Vogelstein, B. Detection and quantification of mutations in the plasma of patients with colorectal tumors. Proc. Nat. Acad. Sci. 2005, 102, 16368-16373.

(12) Perkel, J. Guiding our PCR experiments. BioTechniques 2015, 58, 217-221.

(13) Gingras, I.; Salgado, R.; Ignatiadis, M. Liquid biopsy: will it be the 'magic tool' for monitoring response of solid tumors to anticancer therapies? Curr. Opin. Oncol. 2015, 27, 560-567.

(14) Nicholls, P. J.; Malcolm, A. D. B. Nucleic Acid Analysis by Sandwich Hybridization. J. Clin. Lab. Anal. 1989, 3, 122-135.

(15) Cao, X.; Ye, Y.; Liu, S. Gold nanoparticle-based signal amplification for biosensing. Anal. Biochem. 2011, 417, 1-16.

(16) Esashika, K.; Saiki, T. DNA Hybridization Assay Using Gold Nanoparticles and Electrophoresis Separation Provides $1 \mathrm{pM}$ Sensitivity. Bioconjugate Chem. 2018, 29, 182-189.

(17) Gunnarsson, A.; Sjövall, P.; Höök, F. Liposome-Based Chemical Barcodes for Single Molecule DNA Detection Using Imaging Mass Spectrometry. Nano Lett. 2010, 10, 732-737.

(18) Magde, D.; Rojas, G. E.; Seybold, P. G. Solvent Dependence of the Fluorescence Lifetimes of Xanthene Dyes. Photochem. Photobiol. 1999, 70, 737-744.

(19) Caliendo, A. M.; Gilbert, D. N.; Ginocchio, C. C.; Hanson, K. E.; May, L.; Quinn, T. C.; Tenover, F. C.; Alland, D.; Blaschke, A. J.; Bonomo, R. A.; Carroll, K. C.; Ferraro, M. J.; Hirschhorn, L. R.; Joseph, W. P.; Karchmer, T.; MacIntyre, A. T.; Reller, L. B.; Jackson, A. F.; for the Infectious Diseases Society of America (IDSA). Better tests, better care: improved diagnostics for infectious diseases. Clin. Infect. Dis. 2013, 57, S139-S170.

(20) Edwards, K. A.; Curtis, K. L.; Sailor, J. L.; Baeumner, A. J. Universal liposomes: preparation and usage for the detection of mRNA. Anal. Bioanal. Chem. 2008, 391, 1689-1702.

(21) Lee, M.; Durst, R. A.; Wong, R. B. Development of flowinjection liposome lmmunoanalysis (FILIA) for imazethapyr. Talanta 1998, 46, 851-859.
(22) Baeumner, A. J.; Pretz, J.; Fang, S. A Universal Nucleic Acid Sequence Biosensor with Nanomolar Detection Limits. Anal. Chem. 2004, 76, 888-894.

(23) Lakowicz, J. R. Principles of Fluorescence Spectroscopy; 2nd Ed., Kluwer Academic/Plenum Publishers: New York, London, Moscow, Dordrecht, 1999.

(24) Liu, J.; Liu, C.; He, W. Fluorophores and Their Applications as Molecular Probes in Living Cells. Curr. Org. Chem. 2013, 17, 564579 .

(25) Underhill, H. R.; Kitzman, J. O.; Hellwig, S.; Welker, N. C.; Daza, R.; Baker, D. N.; Gligorich, K. M.; Rostomily, R. C.; Bronner, M. P.; Shendure, J. Fragment Length of Circulating Tumor DNA. PLOS Genet. 2016, 12, No. e1006162.

(26) Shrivastava, A.; Gupta, V. B. Methods for the determination of limit of detection and limit of quantitation of the analytical methods. Chron.Young Sci. 2011, 2, 21-25.

(27) Macanovic, M.; Lachmann, P. J. Measurement of deoxyribonuclease I (DNase) in the serum and urine of systemic lupus erythematosus (SLE)-prone NZB/NZW mice by a new radial enzyme diffusion assay. Clin. Exp. Immunol. 1997, 108, 220-226.

(28) Cogoi, S.; Jakobsen, U.; Pedersen, E. B.; Vogel, S.; Xodo, L. E. Lipid-Modified G4-Decoy Oligonucleotide Anchored to Nanoparticles: Delivery and Bioactivity in Pancreatic Cancer Cells. Sci. Rep. 2016, 6, 38468.

(29) Das, J.; Kelley, S. O. High-Performance Nucleic Acid Sensors for Liquid Biopsy Applications. Angew. Chem., Int. Ed. 2019, DOI: 10.1002/anie.201905005.

(30) Madic, J.; Zocevic, A.; Senlis, V.; et al. Three-color crystal digital PCR. Biomol. Detect. Quantif. 2016, 10, 34-46.

(31) Madic, J.; Jovelet, C.; Lopez, J.; André, B.; Fatien, J.; Miran, I.; Honoré, A.; Lezquita, L.; Besse, B.; Lacroix, L.; Droniou, M. EGFR C797S, EGFR T790M and EGFR sensitizing mutations in non-small cell lung cancer revealed by six-color crystal digital PCR. Oncotarget 2018, 9, 37393.

(32) Woolley, C. F.; Hayes, M. A. Recent developments in emerging microimmunoassays. Bioanalysis 2013, 5, 245-264.

(33) Mehrotra, M.; Singh, R. R.; Loghavi, S.; Duose, D. Y.; Barkoh, B. A.; Behrens, C.; Patel, K. P.; Routbort, M. J.; Kopetz, S.; Broaddus, R. R.; Medeiros, L. J.; Wistuba, I. I.; Luthra, R. Detection of somatic mutations in cell-free DNA in plasma and correlation with overall survival in patients with solid tumors. Oncotarget 2018, 9, 1025910271.

(34) Diamandis, E. P.; Fiala, C. Can circulating tumor DNA be used for direct and early stage cancer detection? F1000Res. 2017, 6, 21292136.

(35) Li, M.; Diehl, F.; Dressman, D.; Vogelstein, B.; Kinzler, K. W. BEAMing up for detection and quantification of rare sequence variants. Nat. Methods 2006, 3, 95-97.

(36) Feuillie, C.; Merheb, M. M.; Gillet, B.; Montagnac, G.; Daniel, I.; Hänni, C. A Novel SERRS Sand-wich-Hybridization Assay to Detect Specific DNA Target. PLoS One 2011, 6, No. e17847.

(37) van den Kieboom, C. H.; van Domburg, T. S. Y.; de Jonge, M. I.; Ferwerda, G.; Hermans, P. W. M. A novel high throughput nucleic acid sandwich hybridization assay on a gold substrate. Anal. Methods 2013, 5, 2835-2839.

(38) Wang, J.; Chen, J.; Sen, S. MicroRNA as biomarkers and diagnostics. J. Cell. Physiol. 2016, 231, 25-30.

(39) Ferrauto, G.; Delli Castelli, D.; Di Gregorio, E.; Terreno, E.; Aime, S. LipoCEST and cellCEST imaging agents: opportunities and challenges. Wiley Interdiscip. Rev. Nanomed. Nanobiotechnol. 2016, 8, 602-618.

(40) Ferrauto, G.; Di Gregorio, E.; Baroni, S.; Aime, S. Frequencyencoded MRI-CEST agents based on paramagnetic liposomes/RBC aggregates. Nano Lett. 2014, 14, 6857-6862.

(41) Mulas, G.; Ferrauto, G.; Dastrù, W.; Anedda, R.; Aime, S.; Terreno, E. Insights on the relaxation of liposomes encapsulating paramagnetic Ln-based complexes. Magn. Reson. Med. 2015, 74, 468473. 
(42) Ferrauto, G.; Carniato, F.; Di Gregorio, E.; Tei, L.; Botta, M.; Aime, S. Large photoacoustic effect enhancement for ICG confined inside MCM-41 mesoporous silica nanoparticles. Nanoscale 2017, 9, 99-103.

(43) Srivastava, K.; Ferrauto, G.; Young, V. G., Jr.; Aime, S.; Pierre, V. C. Eight-Coordinate, Stable Fe(II) Complex as a Dual19F and CEST Contrast Agent for Ratiometric pH Imaging. Inorg. Chem. 2017, 56, 12206-12213.

(44) McHugh, M. L. Multiple comparison analysis testing in ANOVA. Biochem. Med. 2011, 21, 203-209. 